\title{
APPENDICITIS IN PRESCHOOL CHILDREN - A CONTINUING CLINICAL CHALLENGE
}

\author{
Diana Benchia ${ }^{1}$, Klara Sârbu ${ }^{2}$, Ștefan Popa ${ }^{1,3}$, Ioan Sârbu ${ }^{1,3}$, Irene Paula Popa ${ }^{4}$ Carmen Iulia \\ Ciongradi ${ }^{1,3}$
}

\section{Abstract}

Introduction: Acute appendicitis is one of the most common causes of acute abdominal pain in pediatric population. The condition most commonly requiring emergent surgery in children still retains its diagnostic challenges, especially in preschoolers, despite significant advances in therapeutics and medical imaging, due to the variety of symptoms and the rapid development of complications. The purpose of this study is to determine the prevalence of appendicitis in preschool children, the preferred route of management, as well as to comparative evaluate the data obtained from children of other ages, in terms of time from admission to surgery, length of surgery and length of hospital stay. Materials and Methods: Retrospective analysis of children admitted with the diagnose of acute appendicitis at Emergency Children Hospital „Sf. Maria” Iasi between January 2012 and July 2018 and analysis of demographics, preoperative, intraoperative, and postoperative data of three age groups: preschool (less than 6 years of age), school children (6 to 11 years) and teenager (12-17 years). Results: Of 2165 patients selected for this study, 5.35\% patients were preschool children, with a gender distribution not significantly different respect to the other two groups. From 99 patients receiving the final diagnosis of appendicitis in the preschool group, 95 were operated, mainly open (78 cases). The surgery was done in the first day after admission in $59.8 \%$, with a longer duration of surgery, both in open and laparoscopic appendectomy, respect to the other two groups. In the preschool group, the frequency of peritonitis was much higher compared to other ages and the hospital stay was longer. There was no link between a longer time to appendectomy and poorer outcomes. Conclusions: Despite progresses in diagnose and treatment, acute appendicitis in preschool children is a continuing challenge.

Keywords: preschool children, acute appendicitis, peritonitis, laparoscopic surgery

\section{Introduction}

First described in 1886 by Reginald Fitz, acute appendicitis is one of the most common surgical causes of acute abdominal pain in pediatric patients $[1,2]$. The peak incidence is considered between the first and the second decade of life, albeit it is rarely considered in children younger than five years of age [3]. Unlike school-aged and adolescent children, younger children do not exhibit the classic clinical picture of anorexia and peri-umbilical pain that migrates to the right lower quadrant, followed by nausea or vomiting and fever [4].

Despite significant advances in therapeutics and medical imaging, the condition most commonly requiring emergent surgery in children still retains its diagnostic challenges. The non-specific clinical presentation of acute appendicitis in preschool children is thought to be responsible for diagnosis delay and therefore for a higher rate of complications [5].

The purpose of this study is to determine the prevalence of appendicitis in preschool children, the preferred route of management, as well as to evaluate the time from admission to surgery, the length of surgery and hospital stay. Furthermore, the aim is to analyze and compare the results with the data found in the literature.

\section{Material and method}

A retrospective analysis was carried out for all children less than 18 years old with the main diagnosis of acute appendicitis, using the International Classification of Diseases (ICD-10) codes for appendicitis. Between January 2012 and July 2018, a total of 2165 patients admitted at the "Sf. Maria" Emergency Clinical Hospital for Children, Iaşi, Romania with acute appendicitis were charted and retrospectively reviewed. Demographics, preoperative, intraoperative, and postoperative data were collected from patient's medical records and added to Microsoft Excel for further analysis. The patients presented with common symptoms of acute appendicitis, including right lower quadrant pain, fever, anorexia, nausea, and vomiting.

The biological findings, such as leukocytosis and elevated C-reactive protein, were taken to consideration during the diagnosis of the selected patients.

1 „Sfânta Maria” Emergency Children Hospital Iaşi

${ }^{2}$ Klara Sârbu MD Office, Târgu-Neamț, Neamț

${ }^{3}$ „Grigore T. Popa” University of Medicine and Pharmacy Iaşi

${ }^{4}$ „Prof. Dr. George I.M. Georgescu” Institute of Cardiovascular Diseases Iași

E-mail: diana_benchia@yahoo.com; paula.cioban@gmail.com; klarasarbu@yahoo.com; dr_popastefan@yahoo.com; dr.sarbu@gmail.com; iuliaciongradi@yahoo.com 
The particular focus of this study was on the group of preschool children, which were defined by $<6$ years of age. For a better comparison, the rest of the patients were divided into two further groups of age, from $6-11$ and from $12-17$, representing roughly the school children and "teenager".

The age at diagnosis, management, duration of each type of surgery, the duration of hospital stay, and the meantime between the admission and the surgery for each subgroup were obtained and analyzed.

\section{Results}

A total of 2165 patients were selected for this study, based on all inclusion criteria. 116 patients were preschool children, having an age less than 6 years. The group of $6-$ 11 years had 721 patients, while the group $12-17$-year-old ones was represented by 1328 patients. This makes the group of $12-17$ years of age the most represented with a percentage of $61,3 \%$ and the group of preschool children the least represented with a percentage of $5,35 \%$ of all registered cases. (Table I)

When it comes to gender, we observed an almost even distribution, with no statistical significance differences.

In the group of preschool children, 73 patients were diagnosed with appendicitis on admission. The group of 6 to 11 years included 630 patients that were diagnosed as such. The oldest patients, the group of age between 12 and 17 years had 1028 cases of appendicitis, according to the ICD-10 on admission. This led to a distribution of $3.37 \%$ for the age $0-5$ years, $29 \%$ for the age $6-11$ years, and $47.4 \%$ for the age of $12-17$ years.

The diagnose of appendicitis was further divided into 3 subgroups: acute appendicitis with peritoneal abscess, acute appendicitis with generalized peritonitis, noncomplicated appendicitis

In total there were 99 cases with final diagnosis of appendicitis in preschool children. 31 of them had acute appendicitis with peritoneal abscess, 65 patients had acute appendicitis with peritonitis and only 3 had acute appendicitis. The group of 6 to 11 years included 644 with final diagnosis appendicitis, 421 with noncomplicated appendicitis, 159 with acute appendicitis with peritoneal abscess, and 64 with acute appendicitis with peritonitis. The oldest patients, the group of age between 12 and 17 years had 1194 cases of appendicitis, 706 of them had noncomplicated appendicitis, 337 had acute appendicitis with peritoneal abscess and 151 had acute appendicitis with peritonitis (Figure 1).

\begin{tabular}{|c|c|c|}
\hline AGE (y) & NUMBER OF CASES & $\%$ \\
\hline $\mathbf{0 - 5}$ & 116 & $5,35 \%$ \\
\hline $\mathbf{6 - 1 1}$ & 721 & $33,5 \%$ \\
\hline $\mathbf{1 2 - 1 7}$ & 1328 & $61,3 \%$ \\
\hline Total & 2165 & $100,0 \%$ \\
\hline
\end{tabular}

Table I. Number of cases admitted as appendicitis

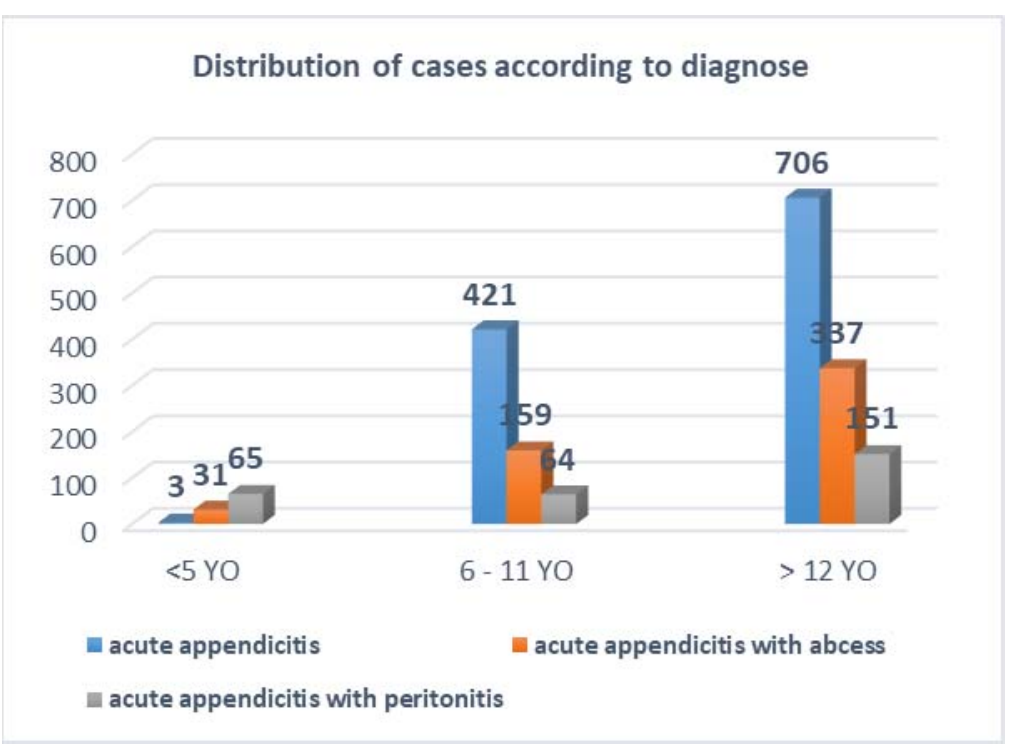

Fig. 1. Distribution of cases according to the type of appendicitis 


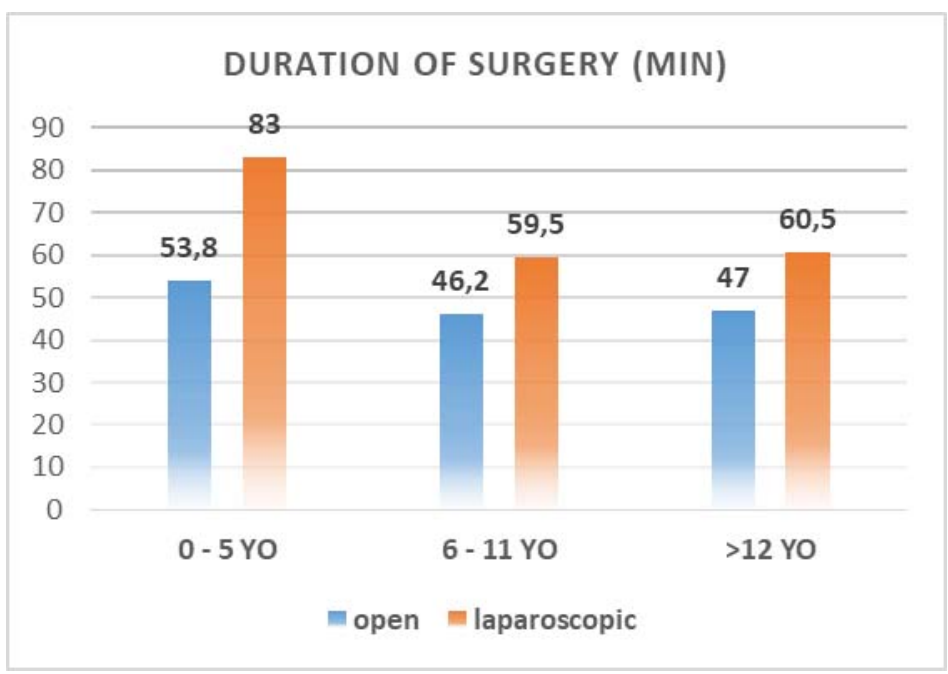

Fig. 2. Duration of surgery

\begin{tabular}{|c|c|c|}
\hline Group of age & Open appendectomy & Laparoscopic appendectomy \\
\hline $\mathbf{0 - 5}$ & 78 & 17 \\
\hline $\mathbf{6 - 1 1}$ & 463 & 114 \\
\hline $\mathbf{1 2 - 1 7}$ & 837 & 229 \\
\hline Total & 1378 & 360 \\
\hline
\end{tabular}

Table II. Type of surgery

\begin{tabular}{|c|c|}
\hline \multirow{2}{*}{ AGE (y) } & MEAN DURATION OF HOSPITALIZATION (d) \\
\hline $0-5$ & $\mathbf{8 , 1 4}$ \\
\hline $6-11$ & $\mathbf{5 , 4 5}$ \\
\hline $12-17$ & $\mathbf{5 , 7 9}$ \\
\hline Total & $\mathbf{5 , 8 1}$ \\
\hline
\end{tabular}

Table III. Mean duration of hospitalization 
Out of a total of 2165 patients included in this study, 1937 had a final diagnosis of appendicitis, and 1738 of them underwent surgery. The surgical interventions were divided into open appendectomy (OA) and laparoscopic appendectomy (LA). All patients with surgical intervention received also medical treatment, consisting mainly of antibiotic coverage.

In the group of preschool children, we had 95 surgical interventions. The vast majority of them were OA with a count of 78 and 17 patients had LA. The group of 6 to 11 years included $463 \mathrm{OA}$ and $114 \mathrm{LA}$. And the group of age between 12 and 17 years had 229 LA and 837 OA (Table II).

Out of 2165 patients from the study, 228 had a different final diagnosis such as gastro-enterocolitis, mesenteric lymphadenitis, Meckel diverticulitis, urinary tract infections, and intussusceptions. Out of 1937 who were diagnosed with appendicitis, 199 received conservative, non-surgical management.

Due to invalid values during data analyze look over, the meantime between the admission and the surgery of the patient, compared first by each group, then focused on the group of preschool children, a certain number of cases had to be excluded. The vast majority of patients (61 out of 95) between $0-5$ years of age were operated on the same day of admission. This represents a distribution of $64,2 \%$ of all patients in this group. 26 patients $(27,3 \%)$ received surgical intervention one day after being admitted to the hospital and 8 patients $(8,5 \%)$ underwent the intervention two or three days after admission.

Considering the duration of surgery, data from only 1153 patients were obtained. We observed that the group of preschool children had the longest mean duration of all groups, as well as in OA as in LA. With a total of 48 minutes operation time in average in OA, the group of 6 11 years old patients and the group of $12-17$ years old were slightly below average with 46,2 minutes (6 - 11 years) and 47 minutes $(12-17$ years). The group of $0-5$ years old on the other hand exceeded clearly the total mean duration, with an average of 53,8 minutes per OA surgery. In LA, the mean duration in $6-11$-year-old patients was with 59,5 minutes, almost similar with the total average time of the group of 12 - 17-year-old with 60,5 minutes, clearly lower than the total average time of 64,8 minutes of surgery, while just like in the case of OA, the meantime of surgery in the patients below 6 years exceeded the total average time with 83 minutes per surgery on average (Figure 2).

A look at the mean duration of hospital stay for the patients involved in this study, shows a higher mean time, with 8,14 days for the group of preschool children than the others. The group of $6-11$ and $12-17$ had a comparable mean duration of hospital stay with 5,45 days for the group of $6-11$ and 5,79 days for the group from $12-17$ years of age. This resulted in a total mean duration of hospital stay of 5,81 days. (Table III)

\section{Discussion}

Acute appendicitis is a relatively uncommon illness in children under the age of six, and it is frequently detected late in this age group [6]. Indeed, appendicitis diagnosis in preschool children is challenging, with a significant probability of misinterpretation due to atypical clinical symptoms and the trivialization of abdominal pain $[7,8]$. The clinical manifestation is frequently variable, and the diagnosis may be misinterpreted by other medical conditions. This observation is consistent with the findings of our study, which show that appendicitis is uncommon in preschool children and peaks during adolescence. In this study, 116 preschool children with suspected appendicitis were involved, with 99 receiving a final diagnosis of appendicitis. Preschool children were in the minority when compared to the other groups, with 12-17-year-olds constituting the vast majority.

The differential diagnosis of acute appendicitis includes gastro-enterocolitis, mesenteric lymphadenitis, Meckel diverticulitis, inflammatory bowel disease, right lower lobe pneumonia, urinary tract infections, and intussusception, particularly in young pre-verbal children [9].

Misdiagnosis in preschool-age children ranges from 19 to 57 percent due to its atypical clinical features, resulting in a high rate of complications $[10,11]$. The high prevalence of complicated appendicitis in young children is due to a combination of factors. The inability of a young child to communicate with his or her parents, as well as atypical presentation and other associated illness, may cause a delay in diagnosis [12-14]. In these young patients, anatomic immaturity and a lack of an adequate omental barrier may contribute to the rapid progression to perforation and peritonitis. Perforation may already be present in 30 percent to 75 percent of children when the diagnosis is made, with young children being at higher risk [13]. This was demonstrated in our study, which found a high rate of appendicular abscess (31\%) and peritonitis $(66 \%)$. Since both, acute appendicitis with peritoneal abscess and acute appendicitis with peritonitis, are defined as complicated appendicitis, this gives us high percentage of complicated appendicitis in the group of preschool children, underlying the importance of this pathology in the particular group of lower age children.

In the pediatric population, complicated intraabdominal infections are most commonly caused by appendix perforation and may be one of the leading causes of morbidity $[15,16]$. A complicated appendicitis usually requires a significantly longer hospital stay. A look at the average length of hospital stay for the patients involved in this study, reveals that the group of preschool children has had a higher mean time, with 8,14 days, than the others $(5,45$ and 5,79$)$.

In terms of surgery duration, we observed that the preschool age group had the longest mean duration of all groups, both in OA and LA, possibly due to complicated appendicitis and anatomical particularities $[15,17]$.

This study also looked at the time it took from presentation to surgery. Despite the fact that the majority of 
patients aged 0 to 5 years were operated on the same day of admission or one day later, there was no link between a longer time to appendectomy and poorer outcomes in terms of total time of surgery procedure and length of hospital stay. Recent studies investigating the time to appendectomy relative to the onset of symptoms found no association, implying that the timing of appendectomy has no effect on adverse event rates, that appendectomy in the middle of the night is no longer justified, and that appendectomy should be considered an elective procedure once antibiotic therapy has been initiated [18-21].

\section{Conclusion}

These study results point out that, despite major breakthroughs in treatments and medical imaging, diagnosing appendicitis in preschool children remains challenging. Despite the low number of incidences, an early diagnosis of acute appendicitis in preschool children is mandatory. It requires a high level of suspicion, a thorough medical history, and periodic physical examinations, due to the variety of symptoms and the rapid development of complications.

Conflicts of Interest: The authors declare no conflict of interest.

\section{References}

1. Fitz RH. Perforating inflammation of the vermiform appendix; with special reference to its early diagnosis and treatment. Am J Med Sci. 1886;92:321-346.

2. Kulik DM, Uleryk EM, Maguire JL. Does this child have appendicitis? A systematic review of clinical prediction rules for children with acute abdominal pain. J Clin Epidemiol. 2013; 66:95-104. doi: 10.1016/j.jclinepi.2012.09.004.

3. Puri P, Boyd E, Guiney EJ, O’Donnell B. Appendix mass in the very young child. J Pediatr Surg. 1981;16:55-57. doi: 10.1016/S0022-3468(81)80115-7.

4. Williams N, Kapila L. Acute appendicitis in the preschool child. Arch Dis Child. 1991;66:1270-1272. doi: 10.1136/adc.66.11.1270.

5. Rothrock SG, Pagane J. Acute appendicitis in children: emergency department diagnosis and management. Ann Emerg Med. 2000; 36:39-51.

6. Almaramhy HH. Acute appendicitis in young children less than 5 years: review article. Ital J Pediatr. 2017;43:15.

7. Pogorelic Z, Domjanovic J, Jukic M, Pericic TP. Acute appendicitis in children younger than five years of age: diagnostic challenge for pediatric surgeons. Surg Infect. 2019;21(3):239.

8. Rothrock SG, Pagane J. Acute appendicitis in children: emergency department diagnosis and management. Ann Emerg Med. 2000;36:39-51.

9. Marzuillo P, Germani C, Krauss BS, Barbi E. Appendicitis in children less than five years old: A challenge for the general practitioner. World J Clin Pediatr. 2015;4:19-24.

10. van den Bogaard VA, Euser SM, van der Ploeg T, de Korte N, Sanders DG, de Winter D, et al. Diagnosing perforated appendicitis in pediatric patients: a new model. J Pediatr Surg. 2016;51:444-8.
11. Bansal S, Banever GT, Karrer FM, Partrick DA. Appendicitis in children less than 5 years old: influence of age on presentation and outcome. Am J Surg. 2012;204:1031-5.

12. Irish MS, Pearl RH, Caty MG, Glick PL. The approach to common abdominal diagnosis in infants and children. Pediatr Clin North Am 1998;45: 729-772.

13. Stefanutti G, Ghirardo V, Gamba P. Inflammatory markers for acute appendicitis in children: are they helpful? J Pediatr Surg 2007;42:773-776.

14. Bansal S, Banever GT, Karrer FM, Partrick DA. Appendicitis in children less than 5 years old: influence of age on presentation and outcome. Am J Surg 2012;204:1031-1035.

15. Naiditch JA, Lautz TB, Daley S, Pierce MC, Reynolds M. The implications of missed opportunities to diagnose appendicitis in children. Acad Emerg Med. 2013;20:592-6.

16. Pogorelic Z, Silov N, Jukic M, Elezovic Baloevic S, Peričić TP, et al. Ertapenem monotherapy versus gentamicin plus metronidazole for perforated appendicitis in pediatric patients. Surg Infect. 2019;20:625-30.

17. van den Boom AL, Gorter RR, van Haard PM, Doornebosch PG, Heij HA, Dawson I. The impact of disease severity, age and surgical approach on the outcome of acute appendicitis in children. Pediatr Surg Int. 2015;31:339-45.

18. Mandeville K, Monuteaux M, Pottker T, Bulloch B. Effects of timing to diagnosis and appendectomy in pediatric appendicitis. Pediatr Emerg Care. 2015;31(11):753-758. http://dx.doi.org/10.1097/PEC.0000000000000596.

19. Boomer LA, Cooper JN, Anandalwar S, et al. Delaying appendectomy does not lead to higher rates of surgical 
site infections: a multi-institutional analysis of children with appendicitis. Ann Surg. 2016;264(1):164-8.

20. Surana R, Quinn F, Puri P. Is it necessary to perform appendectomy in the middle of the night in children? $\mathrm{Br}$ Med J. 1993;306:1168.
21. Yardeni D, Hirschl RB, Drongowski RA, Teitelbaum DH, Geiger JD, Coran AG. Delayed versus immediate surgery in acute appendicitis: do we need to operate during the night? J Pediatr Surg. 2004;39:464-469..

Correspondence to:

\section{Ioan Sârbu}

„Grigore T. Popa” University of Medicine and Pharmacy 16 University Street, 700115

Iaşi, Romania

Phone: +40745760716

Email: Dr.sarbu@gmail.com 